\title{
Hedgehog overexpression leads to the formation of prostate cancer stem cells with metastatic property irrespective of androgen receptor expression in the mouse model
}

Han-Hsin Chang ${ }^{1 \dagger}$, Bo-Yie Chen ${ }^{2 \dagger}$, Chia-Yung Wu' ${ }^{1}$ Zih-Jay Tsao ${ }^{1}$, Ying-Yu Chen ${ }^{1}$, Chin-Pao Chang ${ }^{3}$, Chi-Rei Yang ${ }^{4}$, David Pei-Cheng Lin $2,5,6^{*}$

\begin{abstract}
Background: Hedgehog signalling has been implicated in prostate tumorigenesis in human subjects and mouse models, but its effects on transforming normal basal/stem cells toward malignant cancer stem cells remain poorly understood.

Methods: We produced pCX-shh-IG mice that overexpress Hedgehog protein persistently in adult prostates, allowing for elucidation of the mechanism during prostate cancer initiation and progression. Various markers were used to characterize and confirm the transformation of normal prostate basal/stem cells into malignant cancer stem cells under the influence of Hedgehog overexpression.

Results: The PCX-shh-IG mice developed prostatic intraepithelial neoplasia (PIN) that led to invasive and metastatic prostate cancers within 90 days. The prostate cancer was initiated through activation of $\mathrm{P} 63^{+}$basal/stem cells along with simultaneous activation of Hedgehog signalling members, suggesting that $\mathrm{P} 63^{+} / \mathrm{Patch} 1^{+}$and $\mathrm{P} 63^{+} / \mathrm{Smo}{ }^{+}$ cells may serve as cancer-initiating cells and progress into malignant prostate cancer stem cells (PCSCs). In the hyperplastic lesions and tumors, the progeny of PCSCs differentiated into cells of basal-intermediate and intermediate-luminal characteristics, whereas rare $\mathrm{ChgA}^{+}$neuroendocrine differentiation was seen. Furthermore, in the metastatic loci within lymph nodes, kidneys, and lungs, the P63+ PCSCs formed prostate-like glandular structures, characteristic of the primitive structures during early prostate development. Besides, androgen receptor (AR) expression was detected heterogeneously during tumor progression. The existence of $\mathrm{P} 63^{+} / \mathrm{AR}^{-}, \mathrm{CK} 14^{+} / \mathrm{AR}^{-}$and $\mathrm{CD} 44^{+}$/ $A R^{-}$progeny indicates direct procurement of $A R^{-}$malignant cancer trait.

Conclusions: These data support a cancer stem cell scenario in which Hedgehog signalling plays important roles in transforming normal prostate basal/stem cells into PCSCs and in the progression of PCSCs into metastatic tumor cells.
\end{abstract}

\section{Background}

Adult prostate epithelial stem cells reside within the basal cell layer and possess high self-renewal capacity, leading to the generation of intermediate, luminal, and neuroendocrine cell lineages $[1,2]$. Normally, most of the adult prostate epithelial stem cells differentiate into

\footnotetext{
* Correspondence: pcl@csmu.edu.tw

+ Contributed equally

${ }^{2}$ School of Optometry, Chung Shan Medical University, Taichung 402, Taiwan Full list of author information is available at the end of the article
}

intermediate cells without requirement of androgen receptor (AR) activity. The process is characterized by loss of P63 and gain of CK14 or CD44 expression [3-5]. Then, the intermediate cells undergo terminal differentiation as they form the luminal cells with CK8 expression and become dependent on AR activity for maintenance and fulfilling of their functions [3,4].

Like many other cancer stem cells, a hypothesis of prostate cancer stem cells (PCSCs) originated from normal stem cells has been proposed based on their highly

\section{Biomed Central}


tumorigenic trait and basal/stem cell-like properties of self-renew and differentiation [6-9]. The hypothesis has been supported by some recent studies indicating that androgen-refractory prostate cancer cells contain an apparent basal/stem cell-like signature [9-12], suggesting that these cancer cells may not be derived from the $\mathrm{AR}^{+}$ luminal cell population [9]. In other words, AR signalling may be entirely bypassed during the transformation of prostate stem cells into PCSCs [9]. Alternatively, AR signalling may remain active at early stages of transformation but become repressed as the cancer cells eventually progress into an AR-independent status [13]. The entire bypass pathway has attracted much attention recently, since the basal/stem cells in human prostates are $\mathrm{AR}^{(-}$or low) [14], likely to be the direct origin of androgen-independent cancer cells through tumorigenic transformation, although there has been no evidence so far to support this hypothesis.

Hedgehog (Hh) signalling plays a key role in stem cell plasticity and in many developmental, physiological, and pathogenetic processes [15]. Binding of the Hedgehog ligand to the Patched 1 (Patch1) receptor releases the Patch1-associated Smoothened (Smo) G-protein, which triggers a cascade of intracellular signalling activations that lead to the binding of downstream transcription factors, e.g., Gli1, Gli2 and Gli3 to their target sequences and then expression of target genes involved in the control of cell division or differentiation [16]. Aberrant Hh signalling activation has been implicated in prostate tumorigenesis in human subjects and mouse models [17-22]. Previously, we had confirmed that Hh signalling members are expressed in tumorigenic $\mathrm{P}^{+} 3^{+}$basal cells in human specimens and these cells are capable of differentiation into multiple lineages, suggesting that $\mathrm{Hh}$ signalling may promote primary prostatic cancer stem cells [20]. However, the tumorigenic activation of basal/ stem cells and their progression toward a metastatic status under the influence of Hh signalling remain to be further elucidated. To further characterize the basal cells during tumorigenic activation, we established a mouse prostate cancer model in which prostate tumorigenesis was induced from a normal status through persistent Hh overexpression [21], taking advantage of using mouse models to elucidate tumor formation and evaluate candidate therapeutic agents $[23,24]$.

In this study, we used the Hh overexpression mouse model to elucidate whether the PCSCs arise from $\mathrm{P}^{+}{ }^{+}$ basal/stem cells and to examine whether these cancer cells can maintain stem cell characteristics after metastasis. More importantly, we intended to elucidate whether $\mathrm{P} 63^{+}$basal/stem cells can be directly transformed into $\mathrm{AR}^{-}$cancer cells. We demonstrated that $\mathrm{Hh}$ overexpression initiated malignant transformation of $\mathrm{P} 3^{+}$basal/stem cells that subsequently differentiated into both $\mathrm{AR}^{+}$and $\mathrm{AR}^{-}$progeny of the basal-intermediate and intermediate-luminal progeny, but rare $\mathrm{ChgA}^{+}$ neuroendocrine cells. The Hh-initiated $\mathrm{P} 63^{+}$basal/stem cells were characteristic of PCSCs, as they were able to form primitive prostate-like glandular structures in the metastatic loci. Besides, androgen receptor (AR) expression was detected heterogeneously in PCSCs when they were differentiated into intermediate and luminal cells, indicating that androgen were not necessary for these PCSCs $\left(\mathrm{AR}^{-}\right)$. These data challenge the model of $\mathrm{AR}^{+}$ transition into androgen-independent PCSCs and suggest a potentially better treatment strategy by inhibition of Hedgehog signalling prior to androgen-deprivation therapy.

\section{Methods}

\section{Plasmid vectors}

Mouse Shh-expressing pCX-shh-IG vector and pCX-IG vehicle control vectors were kindly provided by $\mathrm{Dr}$. Kerby C. Oberg, Loma Linda University [25]. The pCXshh-IG vector contains a Shh insert tagged with green fluorescence protein (GFP) sequence driven by a CMV promoter. The vehicle control pCX-IG vector contains the same CMV promoter and the GFP tag, but without the Shh insert. Therefore, presence of GFP in prostates indicated successful introduction of $\mathrm{pCX}$-shh-IG vector and expression of Hh protein.

\section{Intraprostatic injection and electroporation}

ICR strain male mice aged 8-10 weeks were purchased from National Laboratory Animal Center, Academia Sinica, Taipei for use in this study. The mice were anesthetized and exposed of their prostate glands by surgery, followed by intraprostatic injection and electroporation to introduce the PCX-shh-IG or PCX-IG vectors as described in our previous study [21]. All animal procedures were performed following the Guide for the Care and Use of Laboratory Animal that had been promulgated by the Institute of Laboratory Animal Resources and had been approved by the animal care and use committee of Chung Shan Medical University.

\section{Immunohistochemical staining, double-}

\section{immunofluorescence staining and TUNEL assay}

Standard procedures were followed to prepare prostate tissue sections for immunohistochemistry. Antigen retrieval was achieved by boiling tissue in citrate buffer (pH 6.0) for 20 min. Primary antibodies (all at 1:50 dilution) were goat anti-Shh antibody (N-19), rabbit antiPatch1 (H-267), goat anti-Patch1 (G-19), rabbit antiSmo (H-300), rabbit anti-Gli1 (H-300), goat anti-Gli2 (N-20), goat anti-Gli3 (N-19), goat anti-CK14 (C-14), and mouse anti-CD44 (DF-1485); all were purchased from Santa Cruz Biotechnology (Santa Cruz, CA). 
The mouse anti-p63 (4A4), mouse anti-CK8 (TS1), rabbit anti-AR (RB-9030), mouse anti-PCNA (MS-106), rabbit anti-ChgA (RB-9003) and mouse anti-tubulin (MS-581) were purchased from Lab Vision Corporation (Lab Vision, Fremont, CA). The secondary antibodies were horseradish peroxidase- conjugated anti-mouse, anti-goat, and anti-rabbit IgG (all at 1:200) purchased from Jackson ImmunoResearch Laboratories, Inc., PA. For double-immunofluorescence detection, primary antibodies were applied simultaneously, followed by incubation with donkey anti-mouse rhodamine Red-X and FITC, anti-rabbit rhodamine Red-X and FITC anti-goat FITC (1:50) (Jackson ImmunoResearch Laboratories, Inc., PA) and counterstained with DAPI. Standard brightfield and immunofluorescence microscopy were performed for photography using a Zeiss Axioskop2 Plus microscope and SoftWoRx software. In situ apoptosis assay (TUNEL assay) was processed following the manufacturer's instruction (Millipore, Billerica, MA).

\section{Western blot analysis}

Standard procedures were performed for western blot analysis. Briefly, protein extract $(150 \mu \mathrm{g})$ was fractionated on SDS-polyacrylamide electrophoresis gel and transferred to a polyvinylidine difluoride membrane (Immobilon-P, Millipore). The membrane was then incubated with primary antibody (described above) overnight at $4{ }^{\circ} \mathrm{C}$, followed by incubation with secondary antibody (horseradish peroxidase-conjugated antimouse, anti-rabbit, or anti-goat IgG) for 1 hour. The immune complexes on membranes were detected by chemiluminescence methods (ECL, Amersham).

\section{Quantification of positive or double-positive cells}

Tissue sections from five prostates of the pCX-IGinjected vehicle controls and five pCX-shh-IG-injected mouse prostates were used. Each prostate specimen was from an individual mouse. In each specimen, three randomly picked $1000 \mu \mathrm{m}^{2}$ boxes showing the normal, PIN or the CaP sites were used for quantification. The results were presented as the average percentage of double-positive cells over total counted cells. The difference between the normal, PIN, and the CaP sites was analyzed by Student's t test (significant when $\mathrm{p}<0.001$ ).

\section{Results}

\section{Persistent Hedgehog overexpression induced mouse} prostate tumorigenesis

The pCX-shh-IG-injected mouse prostates exhibited discernible tumors on day 90 after the preparation, which was not observed in those of the pCX-IG-injected vehicle controls and the sham injection controls (with $0.9 \%$ $\mathrm{NaCl}$ ). The tumors, found exclusively in the prostates, showed characteristics of progressive tumorigenesis through stages of prostatic gland hyperplasia, prostatic intraepithelial neoplasia (PIN), and prostate cancer (CaP) (Figure 1A). The pCX-shh-IG-injected prostates exhibited strong $\mathrm{Hh}$ protein expression in contrast to the vehicle controls (Figure 1C). Evident Patch1, Smo, Gli1, Gli2, and Gli3 expressions were found in PIN lesions (Figure 2F to 2J) and $\mathrm{CaP}$ (Figure $2 \mathrm{~K}$ to $2 \mathrm{O}$ ) of the pCX-shh-IG-injected prostates, in contrast to the almost absence of expression in the vehicle controls (Figure 2A to 2E), except that few basal cells and stromal cells were $\mathrm{Smo}^{+}$(Figure 2B). The Patch1 and Smo staining patterns were consistent with their membrane localizations (Figure $2 \mathrm{~F}$ to $2 \mathrm{G}$ and $2 \mathrm{~K}$ to $2 \mathrm{~L}$ ), while Gli transcription factors were predominantly detected in the nucleus and cytoplasm (Figure $2 \mathrm{H}$ to $2 \mathrm{~J}$ and $2 \mathrm{M}$ to $2 \mathrm{O}$ ). Moreover, Hh signaling proteins were expressed heterogeneously, i.e. only in some cell lineages of the PIN lesions (Figure 2F to 2J; indicated by arrowheads) and particularly evident in the round-shaped or accumulated basal cells (indicated by arrowheads in the magnified areas of Figure $2 \mathrm{~F}$ to $2 \mathrm{~J}$ ), in contrast to the normal slim and flat basal cells (indicated by arrows in the magnified areas of Figure 2A to 2E). These data strongly suggest that the prostate cancer cells are likely to be transformed from quiescent basal cells under the influence of Hh overexpression. The data were further solidified by immunoblotting assay showing similar results (Figure 2P). Patch1, Smo, Gli1, Gli2 and Gli3 proteins were found highly expressed in the pCX-shh-IG-injected prostates even on day 90 after the preparation, in contrast to the absence or minimal expression in the vehicle controls or sham controls (Figure 2P). Activated forms of Gli2 and Gli3 proteins were dominantly detected in the pCX-shh-IG-injected prostates (Figure 2P; indicated by Gli2-act and Gli3-act), but not in the vehicle and sham controls. Thus, this preparation offers a suitable mouse model to study the effects of persistent $\mathrm{Hh}$ overexpression during prostate cancer initiation and progression.

\section{$\mathrm{P} 63^{+}$basal/stem cells were activated by Hedgehog overexpression}

To understand whether $\mathrm{P} 63^{+}$basal/stem cells were activated under the influence of Hedgehog overexpression, we examined the pCX-shh-IG-injected prostates during the progression of PIN toward $\mathrm{CaP}$ to gain further insights (Figure 3A). The $\mathrm{P} 3^{+}$basal/stem cells in the pCX-shh-IG-injected prostates showed characteristic features of activation, including increased cell density, bigger cell size, disoriented polarity, and displaced localization (Figure 3A; arrow-indicated in (b)). These features are comparable to those observed in $\mathrm{BCH}$ (basal cell hyperplasia) of human prostate specimens [25], in contrast to the few $\mathrm{P} 63^{+}$cells lying flat along the 

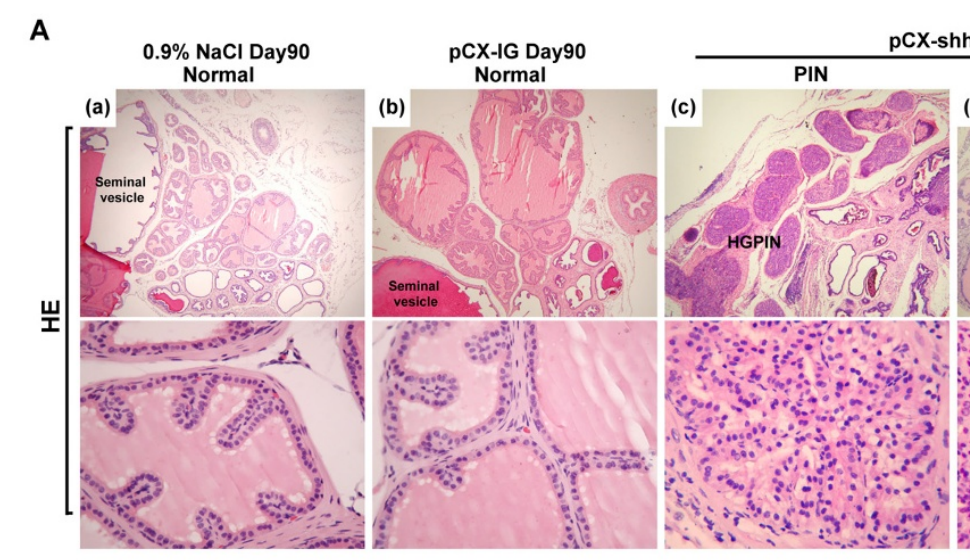

CX-shh-IG Day90
(d) CaP

CaP

C
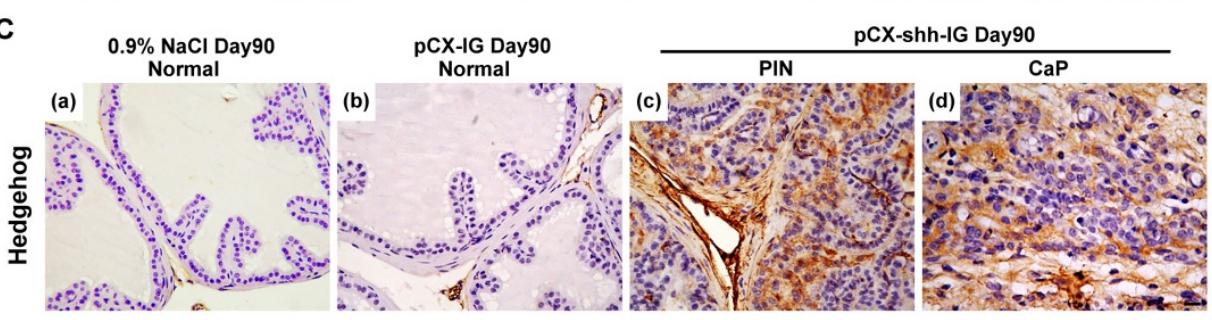

B

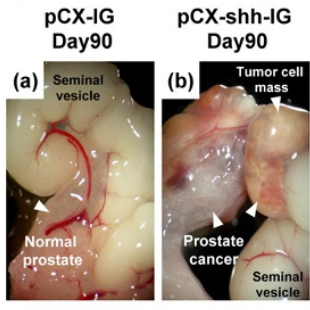

pCX-shh-IG Day90
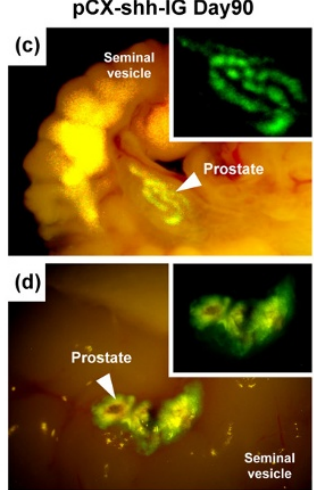

Figure 1 Persistent Hedgehog overexpression induces mouse prostate tumorigenesis. (A) Histopathological analysis with hematoxylineosin stain showing characteristics of progressive tumor formation through stages of PIN and CaP in a pCX-shh-IG-injected prostate at day 90 as compared to sham $(0.9 \% \mathrm{NaCl})$ and vehicle $(\mathrm{pCX}-\mathrm{IG})$ injection controls. The lower pictures are higher magnifications corresponding to the tissue sections shown in the upper pictures. (B) Tumor formation induced by Hedgehog overexpression (arrowheads in (b) indicate tumor mass), as compared to vehicle control (arrowhead in (a)). The prostate gland displayed GFP signals at day 90 after pCX-shh-IG injection (arrowheadindicated in (c) and (d), also magnified in the inlets). (C) The pCX-shh-IG-injected prostate tissue sections were stained strongly for Hedgehog protein in PIN and CaP, in contrast to those of the sham and vehicle controls. Scale bars: $50 \mu m$ in upper (d) of panel A; $10 \mu m$ in lower (d) of panel A and in (d) of panel C. CaP: prostate cancer; HE: hematoxylin-eosin stain; PIN: prostatic intraepithelial neoplasia; HGPIN: high grade prostatic intraepithelial neoplasia.

basement membrane of the vehicle controls (Figure 3A; arrowhead-indicated in (a)). Moreover, apart from nucleus localization in the normal (Figure 3A; arrowheadindicated in (a)) and hyperplasic basal cells (Figure 3A; arrow-indicated in (b)), P63 was detected in the cytoplasm of cells in the HGPIN (high grade PIN) lesions (Figure 3A; arrow-indicated in (c)) and $\mathrm{CaP}$ (Figure 3A; arrowindicated in (d)). Interestingly, P63 was expressed in some but not all populations of prostate cancer cells (Figure 3A; (d)), similar to that observed in human prostate cancers [20].

\section{Hedgehog overexpression promoted $\mathrm{P} 63^{+}$basal/stem cell hyperplasia toward malignant transformation}

Since prostate tumorigenesis was induced and $\mathrm{P} 63^{+}$ basal/stem cells exhibited human $\mathrm{BCH}$-like features of cellular activation in the pCX-shh-IG-injected prostates [20], it is tempting to examine whether Patch1 receptor and its co-receptor Smo are activated in the $\mathrm{P}^{+} 3^{+}$basal/ stem cells. Patch1 and Smo were found highly expressed in the $\mathrm{P} 3^{+}$basal/stem cells of the pCX-shh-IG-injected prostates, being located in the cell membrane of $\mathrm{P} 63^{+}$ basal cells in regions of primary PIN lesions (Figure 3B; arrow in (b) indicated $\mathrm{P} 63^{+} / \mathrm{Patch}^{+}$and arrow in (e) indicated $\mathrm{P} 63^{+} / \mathrm{Smo}^{+}$cells). In contrast, only very limited Patch1 or Smo expression was detected in the quiescent $\mathrm{P} 3^{+}$basal cells in the vehicle controls (Figure 3B; arrowhead-indicated in (a) and (d)). In advanced $\mathrm{CaP}$ lesions, $\mathrm{P} 63^{+}$cancer cells were seen persistently coexpressed with Patch1 or Smo protein and contributed to a certain portion of the heterogeneous cancer cell populations (Figure 3B; arrow in (c) indicated $\mathrm{P} 63^{+} / \mathrm{Patch}^{+}$ and arrow in (f) indicated $\mathrm{P}^{+} 3^{+} / \mathrm{Smo}^{+}$cells). These data support Hedgehog involvement in promotion of basal cell hyperplasia toward malignant transformation.

Metastatic $\mathrm{P} 63^{+}$cancer cells recapitulated prostate-like primitive glandular structure formation in the metastatic loci

To understand whether the $\mathrm{P} 63^{+}$cancer cells induced by Hedgehog overexpression were characteristic of PCSCs, we examined their stemness property and capacity of metastasis. Since pCX-shh-IG vector was only introduced in the prostates, the GFP signals were expected 


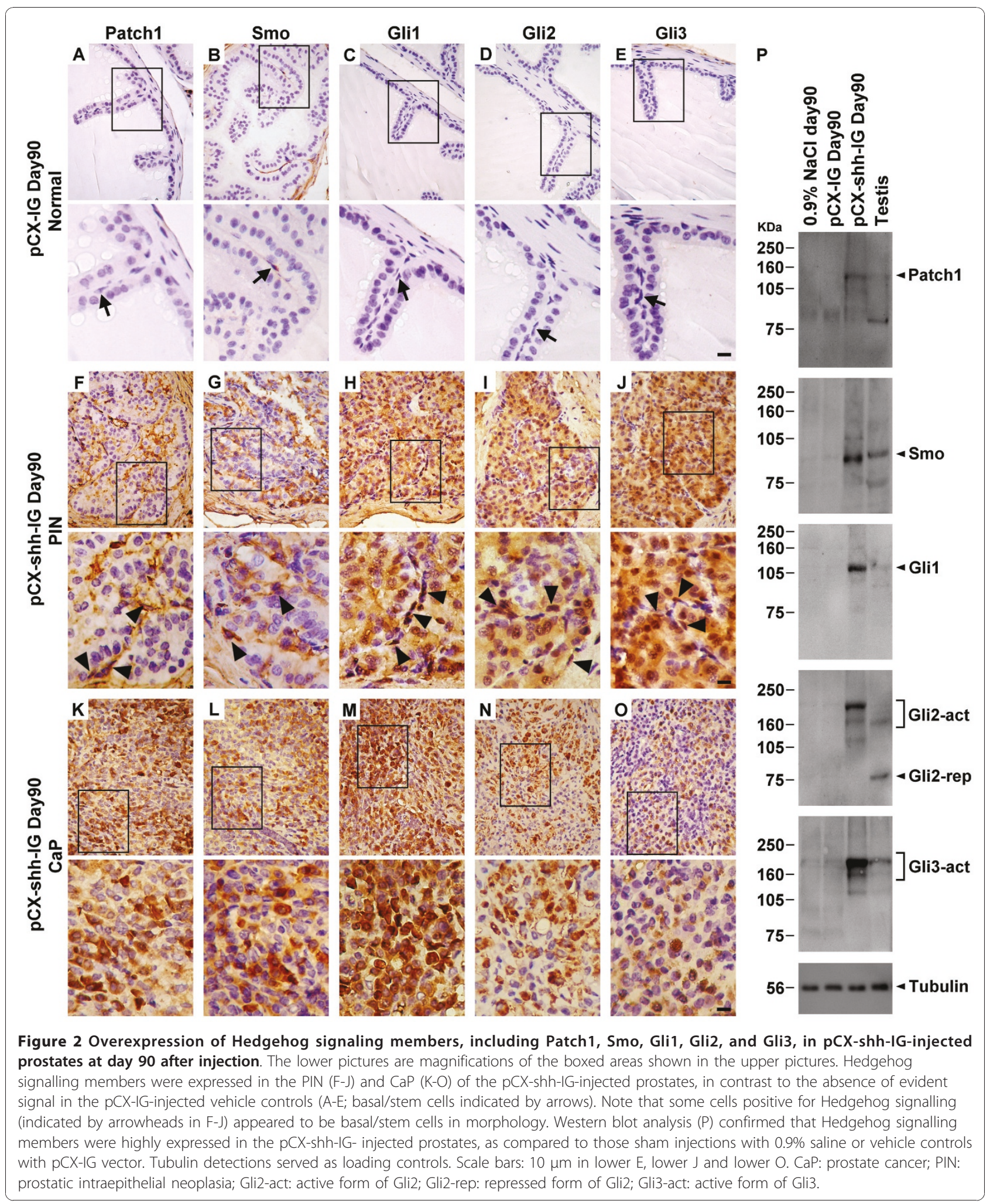




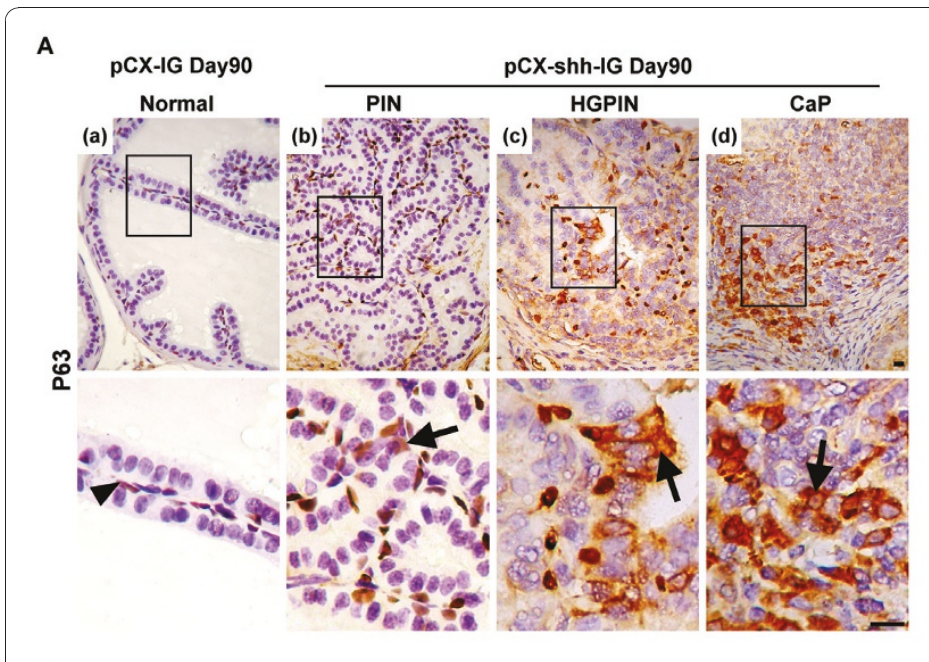

C
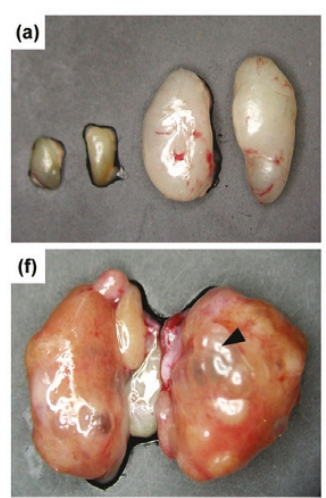

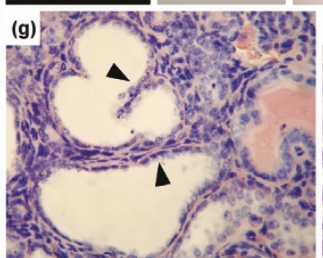

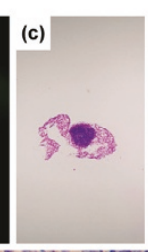
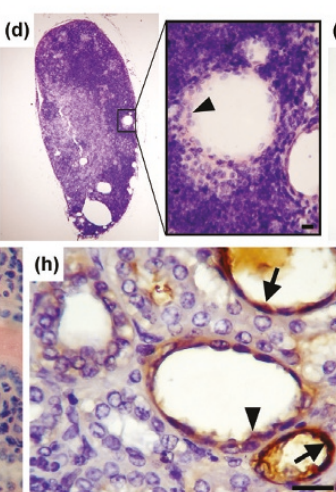

B
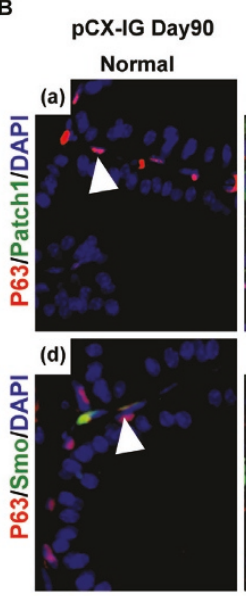
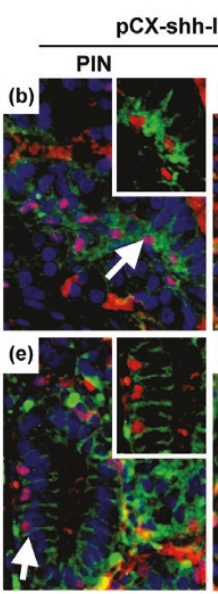
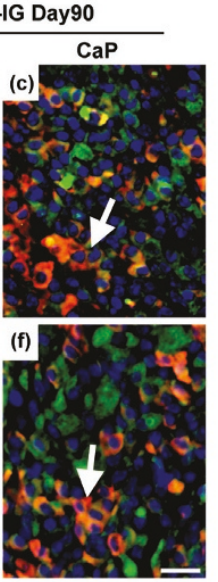

D
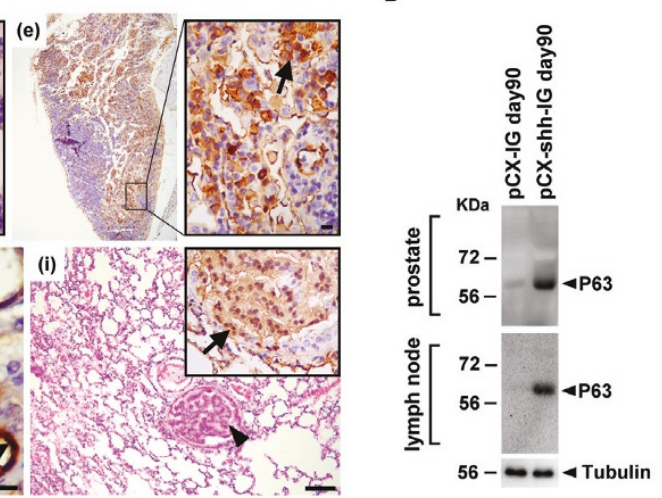

Figure $3 \mathrm{P}_{63^{+}}$basal cells are involved in prostate tumorigenesis and progressed into metastatic cancer cells with Hedgehog overexpression. The boxed areas in (A) and (C) are magnified respectively and inlets in (B) correspond to arrow-indicated areas. (A) Increased ${\mathrm{P} 63^{+}}^{\mathrm{b}}$ basal/stem cell density and altered cellular morphology, including bigger cell size, disoriented polarity, and displaced localization were found along with tumor initiation and progression in the PCX-shh-IG-injected prostate (arrow-indicated in (b), (c) and (d)), in contrast to the normal P63 $3^{+}$cells in the PCX-IG-injected prostate (indicated by arrowhead in (a)). (B) Patch1 and Smo proteins were located within P63 ${ }^{+}$basal cells in the PIN and CaP of pCX-shh-IG-injected prostate. The P63 $/ 3^{+}$atch $1^{+}$and P63 ${ }^{+} / \mathrm{Smo}^{+}$cells are arrow-indicated respectively in (b) and (e), as compared to those in the PCX-IG-injected prostate (arrowhead in (a) and (d)). Patch1 or Smo was co-expressed with P63 ${ }^{+}$in some cancer cells of the advanced prostate cancer (arrow-indicated respectively in (c) and (f)). (C) P63 $3^{+}$cancer cells recapitulated prostate-like glandular structure formation in the metastatic loci (arrowhead-indicated in (d), (f), (g), (h) and (i)). Lymph node metastasis is shown by two enlarged specimens on the right of (a) and in (b), (d), (e), as compared to the normal small lymph node in (c) and the two other small specimens on the left side of (a). Note that GFP signals can be detected in the lymph node in (b). Kidney metastasis is shown in (f-h) and lung metastasis in (i). Arrows in (e), (h), and (i) indicate P63 ${ }^{+}$metastatic cancer cells. (D) Western blot analysis confirmed increased P63 ${ }^{+}$cells in the prostates and the lymph nodes of the pCX-shh-IG-injected mice. All scale bars: $10 \mu \mathrm{m}$. CaP: prostate cancer; PIN: prostatic intraepithelial neoplasia; HGPIN: high grade prostatic intraepithelial neoplasia.

to be detected only within the prostates (Figure 1B; (c) and (d)). Ectopic GFP signals in other organs would indicate prostate cancer cell metastasis (Figure 3C; indicated by arrowhead in (b)). Based on the presence of GFP signals, we found metastasis in the mouse lymph nodes (19/27), kidneys (7/27), and lungs (5/27) following 90 days after introducing PCX-shh-IG vector, but not in the vehicle and sham controls (Figure 3C; (a) indicated two small lymph nodes (on the left) from the sham controls and two enlarged lymph nodes (on the right) from the pCX-shh-IG-injected mice). The metastatic loci of lymph nodes (Figure 3C; (a), (b), (d) and (e)), kidneys (Figure 3C; (f), (g) and (h)), and lungs (Figure 3C; (i)) were infiltrated with $\mathrm{P}^{+} 3^{+}$cells (Figure 3C; indicated by arrows in (e), (h) and (i)). The increase of $\mathrm{P}^{+} 3^{+}$cells in the pCX-shh-IG-injected prostates and lymph nodes after metastasis was confirmed by western blot analysis at day 90 after the preparation (Figure 3D). Furthermore, the $\mathrm{P} 3^{+}$cells within most of the metastatic loci displayed a prostate-like primitive glandular structure (Figure 3C; indicated by arrowheads in (d), (f), (g), (h) and (i)). This finding demonstrated recapitulation of 
prostate formation by the transformed $\mathrm{P}^{+} 3^{+}$basal/stem cells in the metastatic loci. The data evidently demonstrated both cancer cell and stem cell characteristics of the transformed $\mathrm{P} 63^{+}$basal/stem cells, supporting that they could be the origin of PCSCs under the influence of persistent Hedgehog overexpression.

\section{$\mathrm{P}^{+} 3^{+}$basal/stem cells were transformed into $\mathrm{AR}^{+}$or $\mathrm{AR}^{-}$} cancer cells but rarely into $\mathrm{ChgA}^{+}$neuroendocrine cancer cells

Conventional human prostate cancer cells include malignant cells of luminal, basal or neuroendocrine origin at various proportions and unlike the $\mathrm{ChgA}^{-}$luminal cancer cells, tumor cells of neuroendocrine origin are $\mathrm{ChgA}^{+}[26]$. We found only few $\mathrm{ChgA}^{+}$neuroendocrine cells in the PIN lesions and CaP of the pCX-shh-IGinjected prostates (Figure 4A; (b) and (c); indicated by arrowheads), not much different to what was found in the vehicle controls (Figure 4A; (a); indicated by arrowhead). In the pCX-shh-IG-injected prostates, both the $\mathrm{AR}^{+}$and $\mathrm{AR}^{-}$cells were detected, with the $\mathrm{AR}$ protein, if present, located in the nucleus or cytosol in the cells of PIN lesions and CaP (Figure 4A; (e-g)). Wereas, in the vehicle controls, AR was predominantly located in the nucleus of luminal cells (Figure 4A; (d); indicated by arrowhead) and of some basal cells (Figure 4A; (d); arrow (1) indicated $\mathrm{AR}^{+}$basal cells and arrow (2) indicated $\mathrm{AR}^{-}$basal cells). This finding was confirmed by western blot analysis at day 90 after the injection (Figure $4 \mathrm{~A} ;(\mathrm{h}))$. Since cytosolic or no AR protein was observed in some of the cancer cells, it appeared that androgens were not necessarily required for survival of these cells or otherwise they should had been programmed into cell death. To elucidate the situation, we performed TUNEL assay and found no significant apoptosis in PIN lesions and $\mathrm{CaP}$ of pCX-shh-IG-injected prostates (Figure 4B; (b), (c) and (g)), as compared to normal vehicle controls (Figure 4B; (a) and (g)). In contrast, $\mathrm{PCNA}^{+}$ proliferative cells were increased in the PIN and $\mathrm{CaP}$ lesions (Figure 4B; (e), (f) and (h)), but rare in the vehicle controls (Figure 4B; (d) and (h)). These observations indicated that the PCSCs, as induced by persistent

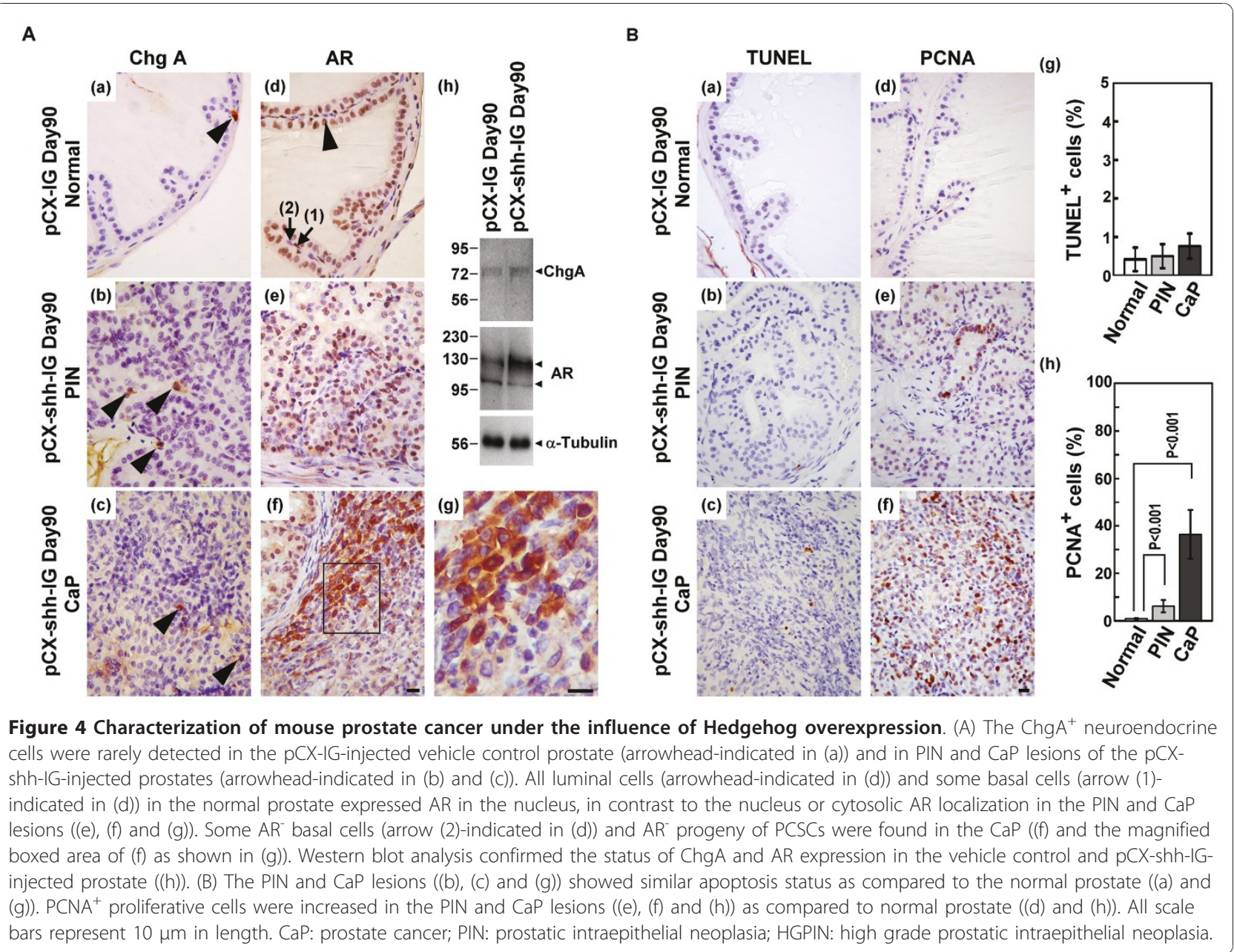


Hedgehog overexpression, did not commit to differentiate into $\mathrm{ChgA}^{+}$neuroendocrine cells and might bypass AR signalling for survival and proliferation.

\section{PCSCs progeny differentiated into basal-intermediate and intermediate-luminal cells}

Since our data had indicated that $\mathrm{ChgA}^{+}$neuroendocrine cells did not constitute the main cellular lineage under the influence of Hh overexpression, the differentiation status of PCSCs was examined with some cell markers, including P63 (primitive basal cells), CK14 (advanced and hyperplastic basal cells), CD44 (intermediate cells), and CK8 (mature luminal cells). In the normal vehicle control prostates, fewer $\mathrm{CK} 14^{+}$basal cells lay flat along the basement membrane (Figure 5A; arrowhead-indicated in (a)), whereas in the PIN and CaP lesions of pCX-shh-IG-injected prostates, CK14 ${ }^{+}$ cells were increased with neoplastic transformation (Figure 5A; arrow-indicated in (b), (c) and (d)). By western blot analysis, we found that CK14, CD44, and CK8 markers were up-regulated in the prostate tumors as compared to the normal prostates (Figure 5B). Double labelling of P63 and CK14 markers showed $\mathrm{P}^{+}{ }^{+} / \mathrm{CK} 14$ (low or -) cells in the normal prostates (Figure 5C; arrowhead-indicated in (a)). In the pCX-shh-IG-injected prostates, as the prostates were induced into PIN and progressed into $\mathrm{CaP}$ status, these $\mathrm{P} 63^{+} / \mathrm{CK} 14^{\text {(low or }-)}$ cells appeared to be differentiated into $\mathrm{P}^{+} 3^{+} / \mathrm{CK}_{14}{ }^{+}$(Figure 5C; arrowhead-indicated in (b)) and further into P63 (low) $/ \mathrm{CK} 14^{+}$(Figure 5C; arrowhead-indicated in (c)) and $\mathrm{P}^{-} / 3^{-} \mathrm{CK}_{14}{ }^{+}$(Figure 5C; arrow-indicated in (b) and (c)) cells. The loss of P63 expression revealed that the PCSCs were differentiated toward the luminal progeny. Comparably, the $\mathrm{CK} 14^{+} / \mathrm{CD} 44^{+}$cancer cells in the PIN and $\mathrm{CaP}$ lesions (Figure 5C; arrow (2)-indicated in (e) and (f)) were likely to be originated from CK14 (low or -)/ CD44 (low or -) cells in the normal prostates (Figure 5C; arrowhead-indicated in (d)) or from CK14 $4^{+} / \mathrm{CD} 44^{\text {(low or -) }}$ cells (Figure 5C; arrow (1)-indicated in (e) and (f)) and CK14 ${ }^{\text {(low or }-)} / \mathrm{CD} 44^{+}$cells (Figure $5 \mathrm{C}$; arrow (3)-indicated in (e) and (f)), as the cells were transformed into PIN and progressed into CaP conditions. Additionally, the CK14 ${ }^{+} / \mathrm{CK}^{+}$cancer cells in the PIN and CaP lesions (Figure $5 \mathrm{C}$; arrow-indicated in (h) and (i)) might be originated from CK14 ${ }^{(\text {low or }-)} / \mathrm{CK}^{-}$cells in the normal prostates (Figure 5C; arrowhead-indicated in (g)), and then differentiated into $\mathrm{CK} 14^{\text {low }} / \mathrm{CK} 8^{\text {(high or }+ \text { ) }}$ progeny (Figure $5 \mathrm{C}$; arrowhead-indicated in (i)). The increase of basal-intermediate $\left(\mathrm{CK}_{14}{ }^{+} / \mathrm{CD} 44^{+}\right)$(Figure 5D) and intermediateluminal $\left(\mathrm{CK}_{14}{ }^{+} / \mathrm{CK}^{+}\right)$(Figure $5 \mathrm{E}$ ) progeny in the PIN and $\mathrm{CaP}$ lesions of pCX-shh-IG-injected prostates were significant when compared to the vehicle controls. The involvement of Hh signalling during PCSCs differentiation in the PIN and CaP lesions was indicated by double labelling of Patch1 and CK14 (Figure 5C; arrow-indicated in (k) and (l)), in contrast to the only few Patch $1^{+}$cells detected in the normal vehicle controls (Figure 5C; arrowhead-indicated in (j)).

\section{The PCSCs progeny differentiation was not totally dependent on androgen-AR axis}

To examine whether malignant differentiation of PCSCs might be independent from the androgen-AR axis in the pCX-shh-IG-injected prostates, we co-localized AR with different markers, including P63, CK14, CK8, CD44, and Patch1 (Figure 5F). Apart from the $\mathrm{AR}^{+}$cells, we detected $\mathrm{P}^{2} 3^{+} / \mathrm{AR}^{-}, \mathrm{CK}_{14}+/ \mathrm{AR}^{-}, \mathrm{CD} 44^{+} / \mathrm{AR}^{-}$and even the $\mathrm{CK}^{+} / \mathrm{AR}^{-}$ cells in the CaP (Figure 5F). The concurrent existence of $\mathrm{AR}^{+}$and $\mathrm{AR}^{-}$cell populations indicated that androgen-AR axis was not indispensably required for PCSCs and their progeny to undergo further differentiation (Figure 5F; (f)). The localization of Patch1 protein in both $\mathrm{AR}^{+}$and $\mathrm{AR}^{-}$ cancer cells (Figure 5F; (e)) revealed the involvement of Hedgehog signalling in both cell lineages.

\section{Discussion}

Basal/stem cells are the origin of all prostate intraglandular cells $[1,27]$ and $\mathrm{P} 63$ has been proposed to be required for prostate stem cell plasticity and differentiation $[27,28]$. Thus, PCSCs are potentially originated from normal $\mathrm{P} 63^{+}$prostate stem cells, although there has been no evidence so far to support this hypothesis. In our previous study analyzing the human specimen [20], we demonstrated that $\mathrm{Hh}$ protein is expressed in $\mathrm{P} 63^{+}$basal cells, in correlation with basal cell hyperplasia and $\mathrm{CaP}$ formation. Our previous data support normal prostate stem cells transformation into PCSCs, although based only on retrospective observations.

In this study, we provide the evidence to further support PCSCs derivation from normal prostate stem cells, based on several lines of observations. Firstly, the entire process of prostate tumorigenesis was reconstituted in vivo and the effects of Hh overexpression on the normal prostate stem cells was shown by the transformation of $\mathrm{P} 63^{+} / \mathrm{Patch}^{(\text {Low or }-)}$ and $\mathrm{P} 63^{+} / \mathrm{Smo}^{\text {(Low or }-)}$ quiescent basal cells into the $\mathrm{P} 63^{+} / \mathrm{Patch} 1^{+}$and $\mathrm{P} 63^{+} / \mathrm{Smo}^{+}$hyperplastic basal cells, comparable to the human $\mathrm{BCH}$ condition that had been previously observed [20]. Secondly, the $\mathrm{P} 63^{+}$cells showed major cancer cell and stem cell peculiarity on the metastatic sites. The $\mathrm{P} 63^{+}$cells were not only present within various metastatic loci, but also differentiated into prostate-like glandular structures where they were located within the basal compartment. Thirdly, the $\mathrm{P} 63^{+}$basal/stem cells, after being transformed into malignant cells, were capable of differentiation into the basal-intermediate $\left(\mathrm{P} 63^{+} / \mathrm{CK} 14^{+}\right)$and intermediate-luminal $\left(\mathrm{CK} 14^{+} / \mathrm{CD} 44^{+}\right.$and $\left.\mathrm{CK} 14^{+} / \mathrm{CK} 8^{+}\right)$ progeny, and rarely the $\mathrm{ChgA}^{+}$neuroendocrine lineage. 
A
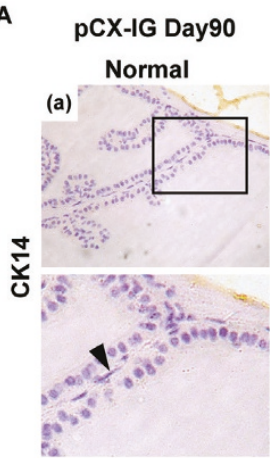

C

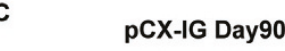

Normal
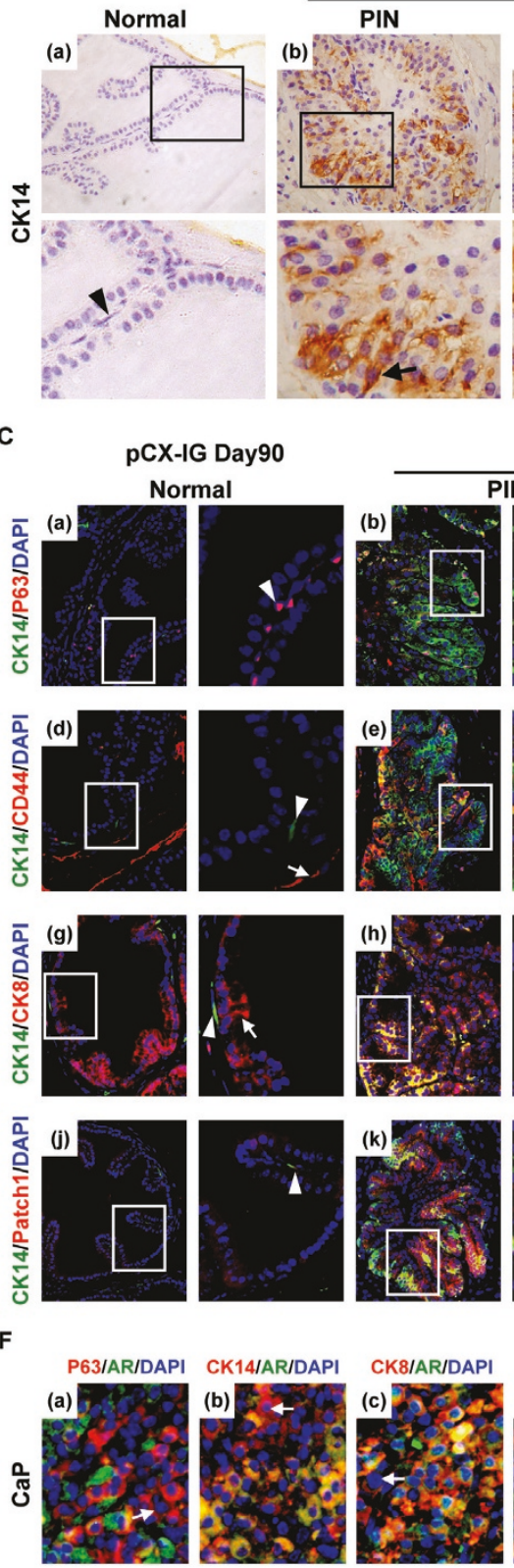

pCX-shh-IG Day90
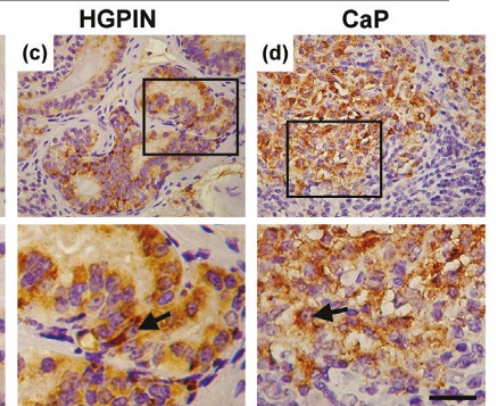

pCX-shh-IG Day90

PIN
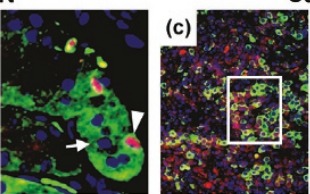

CaP
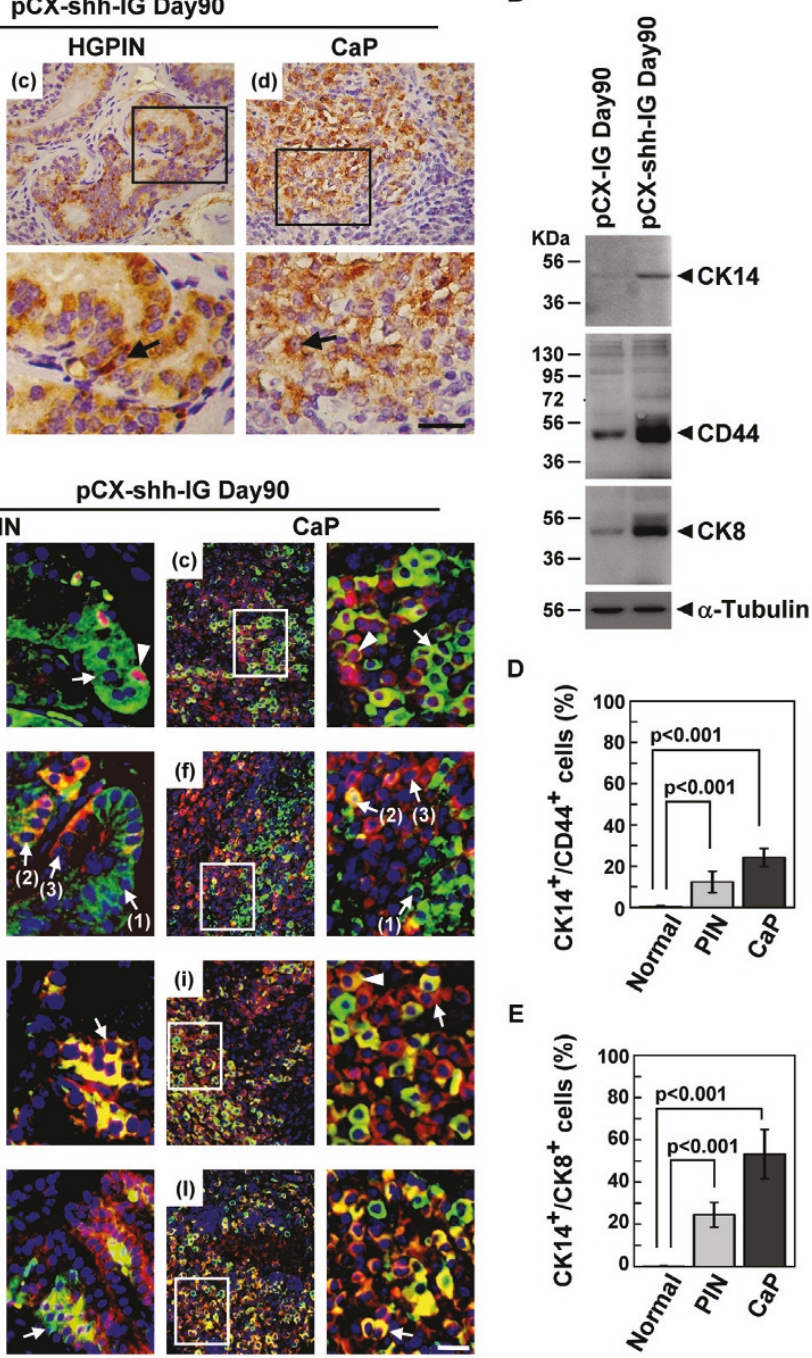

D

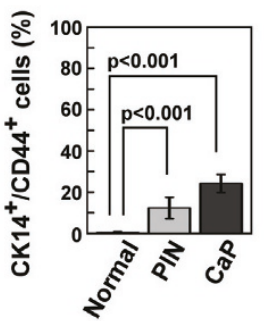

E
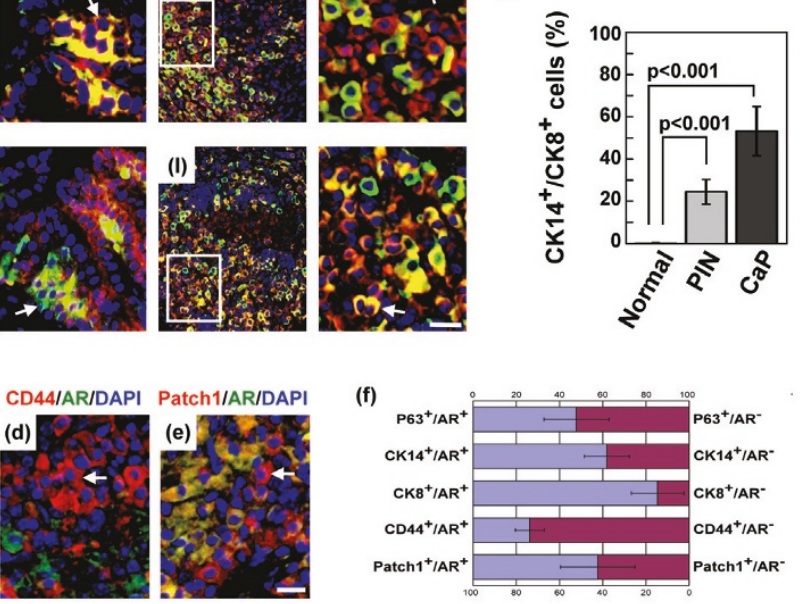

Figure 5 Differentiation status and AR expression profile of PCSCs under the influence of Hedgehog overexpression. The boxed areas in the pictures are further magnified and shown in the corresponding lower or right pictures. (A) Increased CK14 ${ }^{+}$cells along with tumorigenic progression in the PCX-shh-IG-injected prostate ((b), (c) and (d)), as compared to the normal prostate ((a)). (B) Western blot analysis indicated upregulation of CK14, CD44 and CK8 in the pCX-shh-IG-injected prostates as compared to the normal pCX-IG-injected prostates. (C)

Characterization of PCSCs by double-immunofluorescence staining showing differentiation toward CK14 progeny ((a), (b) and (c)), CK14 ${ }^{+}$cells toward CD44 ${ }^{+}$progeny $((\mathrm{d}),(\mathrm{e})$ and $(\mathrm{f}))$, and $\mathrm{CK} 14^{+}$cells toward $\mathrm{CK} 8^{+}$progeny $((\mathrm{g}),(\mathrm{h})$ and $(\mathrm{i}))$. $\mathrm{CK} 14^{+}$differentiation involved Hedgehog signalling activation, as indicated by co-localized Patch1 expression $(\mathrm{j})$, ( $\mathrm{k})$ and $(\mathrm{I})$ ). The basal-intermediate $\left(\mathrm{CK} 14^{+} / \mathrm{CD} 44^{+}\right)$and intermediateluminal $\left(\mathrm{CK}_{1} 4^{+} / \mathrm{CK}^{+}\right)$populations were increased in PIN and CaP of pCX-shh-IG-injected prostates as compared to those of the pCX-IG-injected vehicle controls (D and E). (F) Some PCSCs were AR as indicated by arrows in (a), (b), (c), (d), and (e), even though they were P63 $3^{+}, C K 14^{+}, C K 8^{+}$, $\mathrm{CD}_{4} 4^{+}$, or Patch $1^{+}$. The relative proportions of $\mathrm{AR}^{+}$and $\mathrm{AR}^{-}$cells among P63 ${ }^{+}, \mathrm{CK}_{1} 4^{+}, \mathrm{CK} 8^{+}, \mathrm{CD}_{4} 4^{+}$, and Patch $1^{+}$cell populations were shown respectively in (f). All scale bars represent $10 \mu \mathrm{m}$ in length. CaP: prostate cancer; PIN: prostatic intraepithelial neoplasia; HGPIN: high grade prostatic intraepithelial neoplasia. 
PCSCs derivation from normal prostate stem cells has also been supported by several lines of evidence. Purified cells such as Sca- $1^{+}$and $\mathrm{CD} 49 \mathrm{f}^{+}$cells from the mouse prostates $[29,30]$ or $\mathrm{CD} 44^{+}, \mathrm{CD} 133^{+}$, and $\alpha 2 / \beta 1$ integrin $^{+}$ cells from the human prostates $[31,32]$ were used in renal capsule transplantation studies. These normal cells could generate prostate-like structures in the kidney, supporting the presence of prostate progenitor/stem cells in these purified cell populations and their ability to regenerate in ectopic sites. Such ectopic regeneration capacity has been proven to retain even after the transformation of normal prostate stem cells into PCSCs. For example, $\mathrm{AR}^{-}$P $63^{-} \mathrm{CD} 44^{+} \mathrm{Nestin}^{+}$HPET-5 cells were purified from prostate cancer cell lines [33] and shown to recapitulate prostate-like structures in the mouse kidney after transplantation. Besides, a previous report showed that Hedgehog may recapitulate embryonic gene expression in tumor myofibroblasts [34]. Despite the aforementioned studies, in vivo prostate cancer cell metastasis with a stem cell peculiarity to generate prostate-like structures in the metastatic loci has not been reported. In this study, we demonstrated the infiltration of $\mathrm{P} 63^{+}$cancer cells in the metastatic loci and generation of prostate-like glandular structures. Our data support the prostate cancer stem cell characteristics observed in previous studies and, to our knowledge, these are the first data to confirm that PCSCs metastasis occurs under in vivo conditions.

Clinically, it is known that advanced metastatic androgen-independent prostate cancers exhibit more basal/ stem cell-like differentiation although the underlying mechanism remains unclear $[9,10]$. The transformation of basal/stem cells into PCSCs was proposed as a possible mechanism. In such case, the PCSCs are supposed to maintain high proliferation and differentiation capacities without AR activity [35,36], since androgen is considered not necessary for the survival of basal/stem cells; in contrast to the terminally-differentiated luminal cells which require androgen and AR for survival $[7,9,37]$. Our data showed transition of nuclear to cytoplasmic P63 expression and such transition had been reported to associate with higher proliferative activity, reduced apoptosis, and increased mortality [35]. This may explain the abundant $\mathrm{AR}^{-}$cancer cells found in many high grade prostate cancers [38-40] and the failure of androgen deprivation therapies. Alternatively, persistent AR activation due to loss-of-function mutations has been reported in some androgen-refractory prostate cancers $[13,41]$. Therefore, both $\mathrm{AR}^{+}$and $\mathrm{AR}^{-}$cells were capable of forming androgen-independent prostate cancer cells as the tumorigenesis progresses to the more advanced stages. In this study, both the $\mathrm{AR}^{+}$and $\mathrm{AR}^{-}$ prostate cancer cells were generated under the effects of persistent Hedgehog signalling activation in the mouse model (Figure 4A and Figure 5F). Our data is consistent with the findings of Hedgehog signalling activation in several human prostate cancers [17-20,22], especially in the androgen-independent prostate cancers $[22,42,43]$. Particularly, we showed that some basal/stem cancer cells were $\mathrm{AR}^{-}$, e.g. $\mathrm{P}^{+} 3^{+} / \mathrm{AR}^{-}, \mathrm{CK} 14^{+} / \mathrm{AR}^{-}$and $\mathrm{CD} 44^{+} /$ $\mathrm{AR}^{-}$(Figure 5F; arrowhead-indicated in (a), (b) and (d)). These sub-populations of $\mathrm{AR}^{-}$cancer cells were most likely to contribute directly to the androgen-independent tumors. In fact, androgen ablation by castration could not reduce tumor mass in this model (data not shown). Here, the key findings in this study have confirmed that overexpression of Hedgehog can transform prostate basal cells in vivo and lead the transformed cells to progress into aggressive $\mathrm{AR}^{-} \mathrm{PCSCs}$ progeny. Although the nuclear $\mathrm{AR}^{+}$(active form) and cytoplasmic $\mathrm{AR}^{+}$(inactive form) cells were both observed in the aggressive tumors in this in vivo model and our data showed that Hedgehog signalling activation may substitute the androgen-AR axis for tumor survival or malignant transformation, the underlying mechanisms remain to be further investigated by using in vitro studies.

\section{Conclusions}

Our data support the hypothesis that $\mathrm{P} 63^{+}$hyperplastic basal cells targeted by Hh overexpression may be the true cellular origin of primary prostate cancer. This study also supports that inhibition of Hedgehog signalling may be a better treatment strategy for androgen-independent tumors prior to androgen-deprivation therapy.

\section{Acknowledgements \\ This work was supported by a grant (NSC 96-2321-B-040-007-MY3) to HH Chang and partly by a grant (NSC 97-2321-B-040-004) to DP Lin, from National Science Council, Taiwan. The immunofluorescence microscopy was performed in the Instrument Center of Chung Shan Medical University, which is supported by National Science Council, Ministry of Education and Chung Shan Medical University.}

\section{Author details}

'School of Nutrition, Chung Shan Medical University, Taichung 402, Taiwan. ${ }^{2}$ School of Optometry, Chung Shan Medical University, Taichung 402,

Taiwan. ${ }^{3}$ Division of Urology, Changhua Christian Hospital, Changhua 500,

Taiwan. ${ }^{4}$ Division of Urology, Taichung Veterans General Hospital, Taichung 407, Taiwan. ${ }^{5}$ School of Medical Laboratory and Biotechnology, Chung Shan Medical University, Taichung 402, Taiwan. 'Department of Urology, Chung Shan Medical University Hospital, Taichung 402, Taiwan.

\section{Authors' contributions}

$H H C$, BYC, and DPL designed the study, carried out production of pCX-shhIG and PCX-IG mice, and contributed to the writing of manuscript. CYW helped with animal maintenance, plasmid vector preparation, immunohistochemical and double-immunofluorescence staining. ZJT performed TUNEL assay and western blot analysis. CPC and CRY carried out the quantification of positive and double-positive cells.

\section{Competing interests}

The authors declare that they have no competing interests.

Received: 2 August 2010 Accepted: 18 January 2011

Published: 18 January 2011 


\section{References}

1. Wang Y, Hayward S, Cao M, Thayer K, Cunha G: Cell differentiation lineage in the prostate. Differentiation 2001, 68:270-279.

2. Uzgare $A R, X u Y$, Isaacs JT: In vitro culturing and characteristics of transit amplifying epithelial cells from human prostate tissue. J Cell Biochem 2004, 91:196-205.

3. Tokar EJ, Ancrile BB, Cunha GR, Webber MM: Stem/progenitor and intermediate cell types and the origin of human prostate cancer. Differentiation 2005, 73:463-473.

4. Liu AY, True LD, LaTray L, Nelson PS, Ellis WJ, Vessella RL, Lange PH, Hood L, van den Engh G: Cell-cell interaction in prostate gene regulation and cytodifferentiation. Proc Natl Acad Sci USA 1997, 94:10705-10710

5. Schalken JA, van Leenders G: Cellular and molecular biology of the prostate: stem cell biology. Urology 2003, 62:11-20.

6. Bonkhoff $\mathrm{H}$ : Role of the basal cells in premalignant changes of the human prostate: a stem cell concept for the development of prostate cancer. Eur Urol 1996, 30:201-205.

7. Collins AT, Maitland NJ: Prostate cancer stem cells. Eur J Cancer 2006 42:1213-1218

8. Lawson DA, Witte ON: Stem cells in prostate cancer initiation and progression. J Clin Invest 2007, 117:2044-2050

9. Lang SH, Frame FM, Collins AT: Prostate cancer stem cells. J Pathol 2009, 217:299-306

10. van Leenders GJ, Aalders TW, Hulsbergen-van de Kaa CA, Ruiter DJ, Schalken JA: Expression of basal cell keratins in human prostate cancer metastases and cell lines. J Pathol 2001, 195:563-570.

11. Klarmann GJ, Hurt EM, Mathews LA, Zhang X, Duhagon MA, Mistree T, Thomas SB, Farrar WL: Invasive prostate cancer cells are tumor initiating cells that have a stem cell-like genomic signature. Clin Exp Metastasis 2009, 26:433-446.

12. Patrawala L, Calhoun T, Schneider-Broussard R, Li H, Bhatia B, Tang S, Reilly JG, Chandra D, Zhou J, Claypool K, Coghlan L, Tang DG: Highly purified CD44+ prostate cancer cells from xenograft human tumors are enriched in tumorigenic and metastatic progenitor cells. Oncogene 2006 , 25:1696-1708.

13. Debes JD, Tindall DJ: Mechanisms of androgen-refractory prostate cancer. N Engl J Med 2004, 351:1488-1490.

14. Guzman-Ramirez N, Voller M, Wetterwald A, Germann M, Cross NA, Rentsch CA, Schalken J, Thalmann GN, Cecchini MG: In vitro propagation and characterization of neoplastic stem/progenitor-like cells from human prostate cancer tissue. Prostate 2009, 69:1683-1693.

15. Theunissen JW, de Sauvage FJ: Paracrine Hedgehog signaling in cancer. Cancer Res 2009, 69:6007-6010.

16. Kasper M, Regl G, Frischauf AM, Aberger F: GLI transcription factors: mediators of oncogenic Hedgehog signalling. Eur J Cancer 2006, 42:437-445.

17. Sheng T, Li C, Zhang X, Chi S, He N, Chen K, McCormick F, Gatalica Z, Xie J: Activation of the hedgehog pathway in advanced prostate cancer. $\mathrm{Mol}$ Cancer 2004, 3:29.

18. Sanchez P, Hernandez AM, Stecca B, Kahler AJ, DeGueme AM, Barrett A Beyna M, Datta MW, Datta S, Ruiz i Altaba A: Inhibition of prostate cance proliferation by interference with SONIC HEDGEHOG-GLI1 signaling. Proc Natl Acad Sci USA 2004, 101:12561-12566.

19. Karhadkar SS, Bova GS, Abdallah N, Dhara S, Gardner D, Maitra A, Isaacs JT, Berman DM, Beachy PA: Hedgehog signalling in prostate regeneration, neoplasia and metastasis. Nature 2004, 431:707-712.

20. Chen BY, Liu JY, Chang HH, Chang CP, Lo WY, Kuo WH, Yang CR, Lin DP: Hedgehog is involved in prostate basal cell hyperplasia formation and its progressing towards tumorigenesis. Biochem Biophys Res Commun 2007, 357:1084-1089

21. Chen BY, Lin DP, Liu JY, Chang H, Huang PH, Chen YL, Chang HH: A mouse prostate cancer model induced by Hedgehog overexpression. J Biomed Sci 2006, 13:373-384

22. Azoulay S, Terry S, Chimingqi M, Sirab N, Faucon H, Gil Diez de Medina S, Moutereau S, Maillé P, Soyeux P, Abbou C, Salomon L, Vacherot F, de la Taille A, Loric S, Allory Y: Comparative expression of Hedgehog ligands at different stages of prostate carcinoma progression. J Pathol 2008 216:460-470

23. Sanchez $P$, Clement $V$, Ruiz i Altaba A: Therapeutic targeting of the Hedgehog-GLI pathway in prostate cancer. Cancer Res 2005 65:2990-2992.
24. Stecca B, Mas C, Ruiz i Altaba A: Interference with HH-GLI signaling inhibits prostate cancer. Trends Mol Med 2005, 11:199-203.

25. Oberg KC, Pira CU, Revelli JP, Ratz B, Aguilar-Cordova E, Eichele G: Efficient ectopic gene expression targeting chick mesoderm. Dev Dyn 2002, 224:291-302

26. Bonkhoff $H$, Stein $U$, Remberger $K$ : Endocrine-paracrine cell types in the prostate and prostatic adenocarcinoma are postmitotic cells. Hum Pathol 1995, 26:167-170.

27. Signoretti S, Pires MM, Lindauer M, Horner JW, Grisanzio C, Dhar S, Majumder P, McKeon F, Kantoff PW, Sellers WR, Loda M: p63 regulates commitment to the prostate cell lineage. Proc Natl Acad Sci USA 2005, 102:11355-11360.

28. Kurita T, Medina RT, Mills AA, Cunha GR: Role of p63 and basal cells in the prostate. Development 2004, 131:4955-4964.

29. Burger PE, Xiong X, Coetzee S, Salm SN, Moscatelli D, Goto K, Wilson EL: Sca-1 expression identifies stem cells in the proximal region of prostatic ducts with high capacity to reconstitute prostatic tissue. Proc Natl Acad Sci USA 2005, 102:7180-7185.

30. Lawson DA, Xin L, Lukacs RU, Cheng D, Witte ON: Isolation and functional characterization of murine prostate stem cells. Proc Natl Acad Sci USA 2007, 104:181-186.

31. Xin L, Lawson DA, Witte ON: The Sca-1 cell surface marker enriches for a prostate-regenerating cell subpopulation that can initiate prostate tumorigenesis. Proc Natl Acad Sci USA 2005, 102:6942-6947.

32. Richardson GD, Robson CN, Lang SH, Neal DE, Maitland NJ, Collins AT: CD133, a novel marker for human prostatic epithelial stem cells. J Cell Sci 2004, 117:3539-3545.

33. Gu G, Yuan J, Wills M, Kasper S: Prostate cancer cells with stem cell characteristics reconstitute the original human tumor in vivo. Cancer Res 2007, 67:4807-4815.

34. Shaw A, Gipp J, Bushman W: The Sonic Hedgehog pathway stimulates prostate tumor growth by paracrine signaling and recapitulates embryonic gene expression in tumor myofibroblasts. Oncogene 2009, 28(50):4480-90.

35. Dhillon PK, Barry M, Stampfer MJ, Perner S, Fiorentino M, Fomari A, Ma J, Fleet J, Kurth T, Rubin MA, Mucci LA: Aberrant cytoplasmic expression of p63 and prostate cancer mortality. Cancer Epidemiol Biomarkers Prev 2009, 18:595-600.

36. Kelly K, Yin JJ: Prostate cancer and metastasis initiating stem cells. Cell Res 2008, 18:528-537.

37. Collins AT, Berry PA, Hyde C, Stower MJ, Maitland NJ: Prospective identification of tumorigenic prostate cancer stem cells. Cancer Res 2005, 65:10946-10951.

38. Miyamoto KK, McSherry SA, Dent GA, Sar M, Wilson EM, French FS, Sharief $Y$, Mohler JL: Immunohistochemistry of the androgen receptor in human benign and malignant prostate tissue. J Urol 1993, 149:1015-1019.

39. Chodak GW, Kranc DM, Puy LA, Takeda H, Johnson K, Chang C: Nuclear localization of androgen receptor in heterogeneous samples of normal, hyperplastic and neoplastic human prostate. J Urol 1992, 147:798-803.

40. Huang J, Yao JL, di Sant'Agnese PA, Yang Q, Bourne PA, Na Y: Immunohistochemical characterization of neuroendocrine cells in prostate cancer. Prostate 2006, 66:1399-1406.

41. Yuan X, Balk SP: Mechanisms mediating androgen receptor reactivation after castration. Urol Oncol 2009, 27:36-41.

42. Chen M, Tanner M, Levine AC, Levina E, Ohouo P, Buttyan R: Androgenic regulation of hedgehog signalling pathway components in prostate cancer cells. Cell Cycle 2009, 8:149-157.

43. Shaw G, Price AM, Ktori E, Bisson I, Purkis PE, McFaul S, Oliver RT, Prowse DM: Hedgehog signalling in androgen independent prostate cancer. Eur Urol 2008, 54:1333-1343.

doi:10.1186/1423-0127-18-6

Cite this article as: Chang et al.: Hedgehog overexpression leads to the formation of prostate cancer stem cells with metastatic property irrespective of androgen receptor expression in the mouse model. Journal of Biomedical Science 2011 18:6. 